\title{
FOX, Current State and Possibilities
}

\author{
Radovan Černý ${ }^{1, *}$, Vincent Favre-Nicolin ${ }^{2,3}$, Jan Rohlíček ${ }^{4}$ and Michal Hušák ${ }^{5}$ \\ 1 Laboratory of Crystallography, DQMP, University of Geneva, 24 quai Ernest-Ansermet, \\ CH-1211 Geneva, Switzerland \\ 2 ESRF-The European Synchrotron Radiation Facility, F-38043 Grenoble, France; favre@esrf.fr \\ 3 Université Grenoble-Alpes, CEA/INAC, F-38041 Grenoble, France \\ 4 Department of Structural Analysis, Institute of Physics of the Academy of Sciences of the Czech Republic, \\ Na Slovance 2, 18221 Praha, Czech Republic; rohlicek@fzu.cz \\ 5 Department of Solid State Chemistry, UCT Prague, Technicka 5, 16628 Prague, Czech Republic; \\ Michal.Husak@vscht.cz \\ * Correspondence: Radovan.Cerny@unige.ch; Tel.: +41-022-379-6450 \\ Academic Editors: Silvina Pagola and Shujun Zhang \\ Received: 15 August 2017; Accepted: 13 October 2017; Published: 24 October 2017
}

\begin{abstract}
FOX (Free Objects for Xtallography) is a computer program for solving crystal structures of all types of compounds using the powder data (but also the single crystal data) measured using $X$-ray, neutron and electron diffraction. It works in direct space using the reversed Monte Carlo algorithm of global optimization. Since its release fifteen years ago, it has developed into a powerful tool, simplifying the powder pattern analysis starting from the background determination, indexing and space group selection over the structure modelling using various pre-programmed structural fragments up to the validation of the proposed structural model.
\end{abstract}

Keywords: powder diffraction; crystal structure solution; global optimization; Reversed Monte Carlo; simulated annealing

\section{Introduction}

FOX, 'Free Objects for Xtallography' is a free, open-source program for windows and Linux written for ab-initio structure determination from powder diffraction. It performs direct-space structure determination, allowing a modular description of the structure as a combination of atoms, polyhedra and molecules. Since the first release of FOX in 2001 [1], many features have been added in order to enhance all steps required for a successful structure determination from powder diffraction (SDPD), along with various useful tools to explore crystal structures and powder diffraction patterns. Some of the developments have been reported in previous publications on FOX [2-6]. Here, we complete the information on existing and new features, and give two examples of solving inorganic and organic crystal structures assisted with specific FOX tools.

\section{New Features in FOX}

\subsection{Preparation of Powder Diffraction Data}

In powder diffraction, it is essential to correctly describe the reflection profiles, as well as the background, in order to focus the algorithm on finding the correct crystal structure. Since 2007, FOX has been using the Bayesian approach to give an initial estimate for the background, using the technique described in [7]. The background is automatically estimated, firstly using a linear interpolation, followed by a spline fitting.

The next step-and often a limiting one-is the indexing of the crystalline phase. As shown in Figure 1, it is possible to search for peaks in the diffraction pattern, and then index using the volume 
dichotomy algorithm [8-10], now implemented in Fox. This allows automatic searching for cubic to monoclinic systems, with the volume range being estimated from the observed peaks. Centred systems, as well as spurious peaks, are also taken into account. The indexing of triclinic systems is also possible, as the speed of finding the triclinic lattice is improved considerably. The final results are listed using the standard $\mathrm{M}_{20}$ figure of merit [11]. The indexing of powder patterns measured on multiphase samples is possible using the "decomposition-aided indexing" [12]: the in-situ powder diffraction data are recorded while the sample is heated up to the disappearing of the peaks of one unknown phase (decomposition, melting, reaction). This allows the identification of the peaks corresponding to the disappearing phase in the initial powder pattern.

While FOX relies on integrated profiles [1], and therefore uses figures of merit less sensitive to the exact reflection profile, it is nevertheless better to correctly refine the profiles, especially for large unit cells with significant overlaps between neighbouring reflections. A Le Bail extraction [13] associated with a least-squares optimization is available, with a procedure allowing progressive optimization of more parameters (profile width, asymmetry, unit cell, background, ... ), making the procedure for yielding the best possible profile from the result of the indexing and the initial background estimate almost automatic. This profile-fitting step also extracts the structure factors, which can be used to display Fourier maps during or after structure solution.

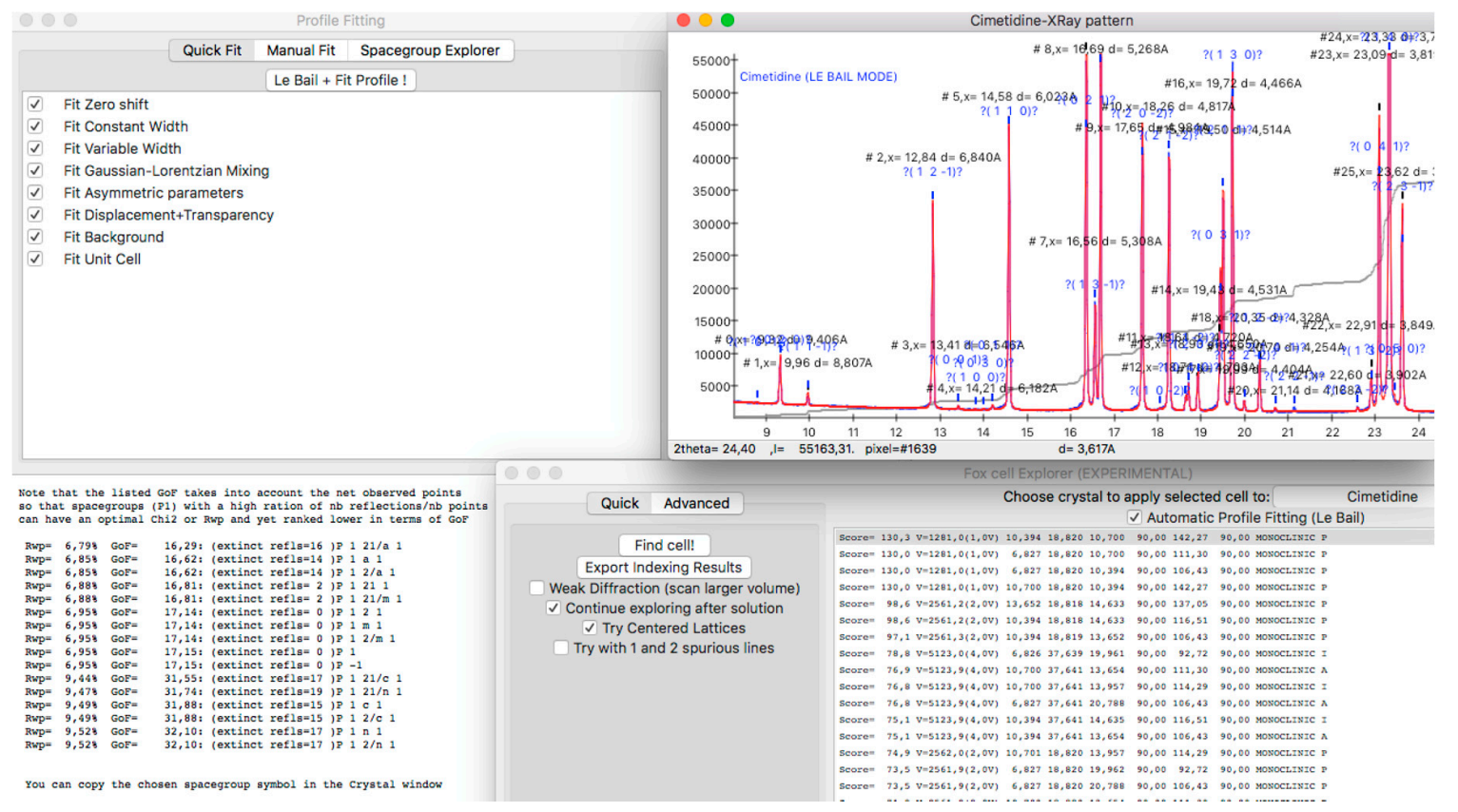

Figure 1. FOX 2017.2 indexing and profile fitting interface. Top left: profile fitting widget, allowing to select groups of parameters to optimize using Le Bail and full-profile least squares. Bottom left: result of the space group explorer, where all space groups compatible with the unit cell are tested and listed with increasing Goodness-of-Fit. The correct choice in this case is the first one, with the highest number of systematic extinctions. Top right: zoomed portion of the observed and calculated powder pattern in profile fitting (Le Bail) mode, with the list of peaks found and predicted indexing. Bottom right: results from a 'quick' indexing, with solutions listed by decreasing $\mathrm{M}_{20}$ score.

Another important step is the determination of the space group. While optimizing the atomic positions in $P 1$ is possible, it greatly increases the number of Degrees of Freedom (DoF), and therefore the time required to solve the structure. A 'space group explorer' widget is thus available, and follows the following protocol: given the unit cell, all possible space group settings starting from $P 1$ are tested using least squares profile fitting, and the $\chi^{2}[14]$ as well as the number of systematic extinctions is recorded for each space group. The results (see Figure 1) are finally listed by increasing values of 
$\chi^{2}$, and the most likely choice usually corresponds to one that has a low $\chi^{2}$ with a high number of extinctions. This systematic approach is akin (though less sophisticated) to the one proposed by [15], but does not provide a single figure-of-merit (the likelihood in [15]) that includes information on both the quality of the fit and the number of extinctions.

Finally, it should be noted that a variety of diffraction data can be taken into account separately or jointly in a single optimization:

- $\quad$ Neutron, X-ray and electron data

- Monochromatic or X-ray tube

- $\quad$ Time-of-flight neutron data (double-exponential pseudo-Voigt profile, [16])

- Textured data: Anisotropic texture using either the March-Dollase model [17], or an Ellipsoid description (courtesy of Aleksander Zaloga).

\subsection{Global Optimization Features}

The core of FOX is optimized for structure solution using a parallel tempering approach [18,19], which allows structures to be found using an ergodic approach, without requiring any algorithm parameters except the number of trials to be tuned. This is coupled with optimized code, which allows evaluation of from $10^{4}$ to $5 \times 10^{4}$ trials/s for 20 independent atoms and 100 reflections, depending on the space group (numbers given using a single $2.5 \mathrm{GHz}$ Intel i7 processor core), i.e., typically a million trial configurations per minute for simple structures.

However, with increasingly complex structures, various strategies have been implemented to improve the likelihood of finding the true structure:

The first-trivial—strategy is to perform multiple optimizations (typically 10-20), each starting from a randomly generated conformation of the structural model, and comparing the results. This can be done automatically, and not only allows exploration of more solutions, but gives a greater confidence in the final solution, as it is obtained several times by the algorithm. This strategy has already been proposed in other programs, such as DASH [20].

Another improvement is to use a least-squares optimization of the crystal structure; during the parallel tempering approach, all structural parameters are modified so that atoms randomly move with amplitudes typically ranging from 0.01 to $10 \AA$. This approach allows efficient exploration of the parameter space, but not being a downhill method, is unlikely to yield the absolute minimum, which can be extremely narrow. Several strategies have been proposed to better exploit the local gradient of the $\chi^{2}$ hypersurface in order to find the solution, such as hybrid Monte-Carlo [21,22] or a Lamarckian approach combined to genetic algorithms [23]. In FOX, it is now possible to perform automatic least-squares refinements, either at the end of a parallel tempering run, or every $150 \times 10^{3}$ trials. This allows the speed of convergence to be greatly increased; e.g., for cimetidine [24], the average number of trials for solving the structure diminishes from $1.6 \times 10^{6}$ to $6 \times 10^{5}$, while the number of least-squares steps only marginally increases the average number of trials per second. This was measured by performing 100 runs of 5 million trials with and without automatic least-squares every $150 \times 10^{5}$ trials, by stopping the optimization when the log-likelihood falls below a cut-off value, guaranteeing that the proximity to the global minimum was certain. Finally, it was also suggested [25] that it would be possible to solve structures by a performing a large number of downhill minimizations from random starting structures-this experimental approach is also possible in FOX by enabling automatic least-squares with a small number of trials per run.

One of the most difficult cases for structure solution includes molecules with flexible cycles (with more than 7 atoms). In this case, the usual approaches to modifying the structure conformation-either using a Z-matrix or using an analysis of bond length and angle restraints [2] — cannot be used; it is impossible to alter the atomic positions through a simple rotation around a given bond inside a cycle without breaking the other bonds of the cycle. The only efficient way to alter the conformation of the atoms in the cycle is to use molecular dynamics (MD); in FOX, this is implemented in the following way: each Molecule object can be attributed a MD energy (which only takes into account the restraints 
of bond lengths and angles), as well as a frequency with which MD moves will be tried. When such a move is tested, atoms in the molecule are given a random initial speed, taking into account the total available MD energy, and the atoms are moved with an adaptive number of steps, depending on the desired average atomic displacement. This allows a random change in the conformation of flexible cycles, without breaking the restraints. The only drawback of this approach is that it is time-consuming, and can increase the average calculation per trial, typically by $20 \%$ to $50 \%$.

In order to solve structures with large unit cells from powder diffraction, the use of differential thermal expansion has been proposed to separate reflections using data collected at different temperatures [26]. In FOX, it is now possible to use several powder patterns with different unit cells, but corresponding to the same crystal (i.e., the same fractional coordinates for the atoms). This enables structures to be optimized by exploiting differential thermal expansion, but without requiring the extraction of the structure factors.

\subsection{Distributed Computing}

An extension with simple grid-computing capabilities (FOXGrid) has been added [6]. This allows the use of the full processing power of the working PC, or adding other computers in the network to the computation process. It is embedded directly in the FOX program, and no additional software is required. The code works so that one instance (the server) is sending jobs to multiple FOX instances (clients) running on computers in the network or on other cores of the same multi-core computer, see Figure 2. When the job is solved, the clients send the result back to the server and request new work; therefore, the structure is solved in parallel. It is clear that adding more computing units to the calculation process further accelerates the structure solution process.

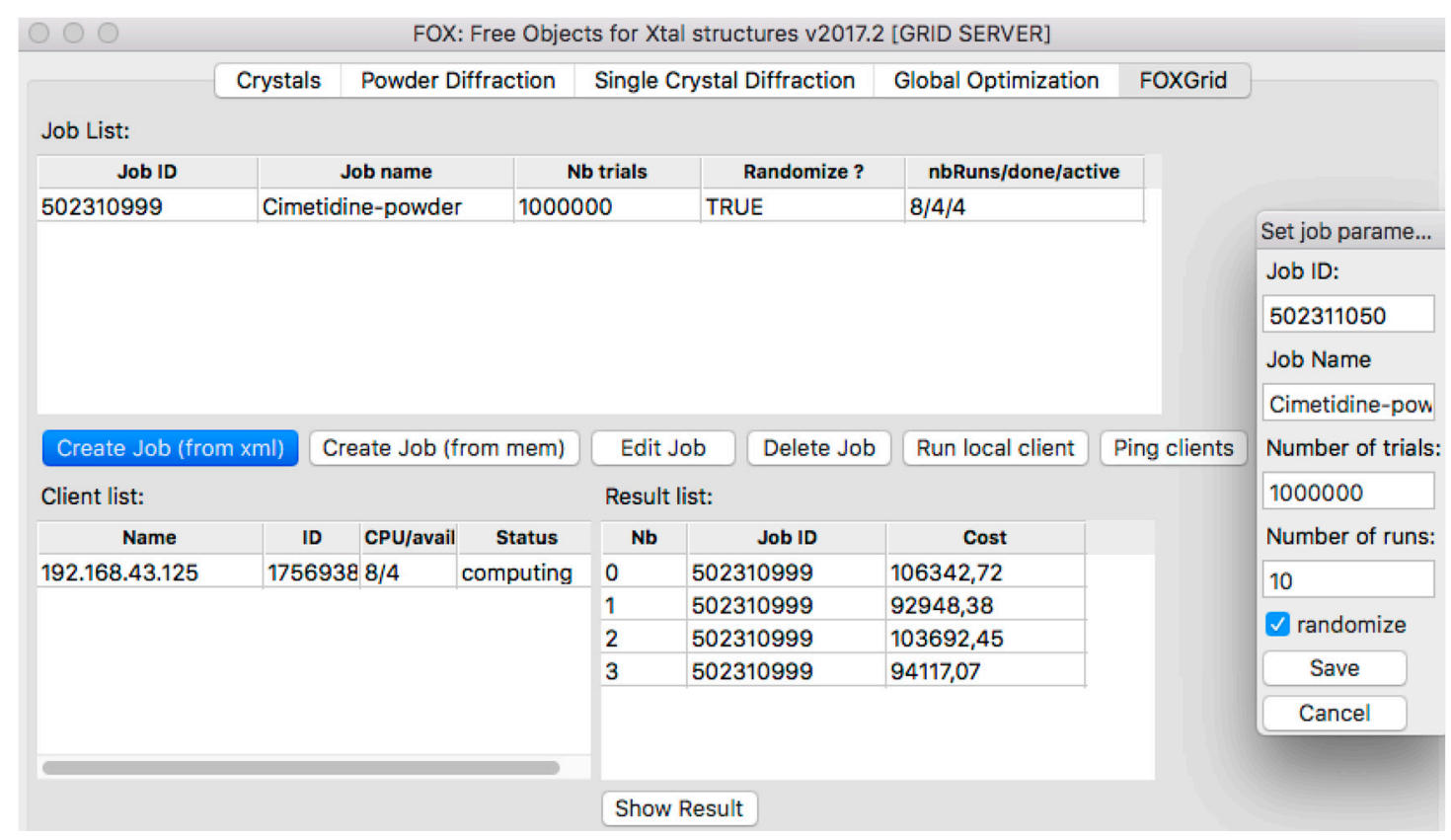

Figure 2. FOXGrid server interface, giving access to distributed computing options. In this example, a single job has been loaded, with 4 runs done out of 8 . Each run starts from a randomized structure, for one million trials. In this case, only the local computer is used, with 4 computing units to exploit the 4 cores of the computer. The results already obtained are listed at the bottom right (and, in this case, are all correct solutions), and can be individually displayed. The inset widget on the right shows the simple interface for creating a new job.

This approach allows solving more complex crystal structures in less time, as well as using the brute-force approach by defining a list of slightly different jobs, e.g., to test several possible space groups 
or different content of the asymmetric part of the unit cell. However, it is important to note at this point that correctly defining the problem at the beginning (correct description of the structure, unit cell and space group) is much more efficient than adding a large number of computing units.

A similar approach was previously reported for the commercial software DASH [20], with its grid module GDASH [27], and was also reported using a cloud computing approach [28].

\subsection{Import and Export, CIF and Crystallography Open Database}

Since the first version, FOX has improved its support for the Crystallographic Information File (CIF) format; it is possible to load both crystal structures and diffraction data (single crystal and powder patterns) from CIF files. Note that, since the wavelength is not listed in powder pattern CIFs, the wavelength loaded in the previous CIF loaded is used instead.

Crystal structures can be exported from FOX as CIF files, in order to be used in other software. It is also possible to export files (crystal description and powder pattern) directly for the FULLPROF software [29].

One additional feature of FOX is the ability to query the Crystallographic Open Database (COD) [30] by giving constraints like a name or keyword, name of authors, list and number of elements, unit cell volume. The results (limited to the first 100 to avoid saturating the database server) can be listed as shown in Figure 3, and double-clicking on the crystal formula will load the CIF file from the COD. Note that this functionality can be limited for computers running behind a firewall, as access to the MySQL port (3306) of the COD server is required.

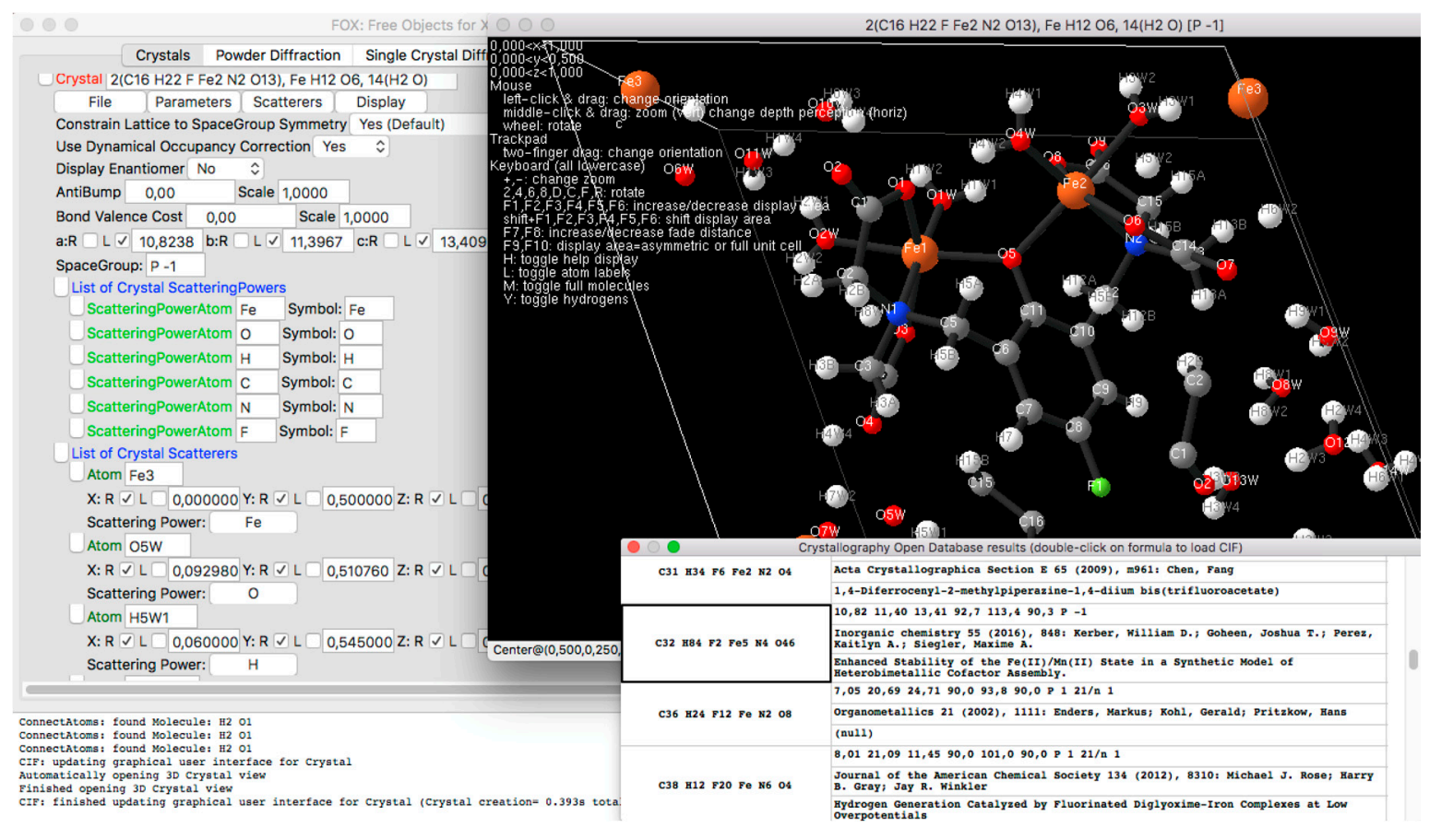

Figure 3. Main FOX interface. Left: main window with the beginning of the description of a crystal structure. Top right: 3D view of the structure, with the help text, showing only the asymmetric unit. Several shortcuts are available to easily toggle between different views (full unit cell, toggle hydrogens, fade atoms outside display volume ... ). Bottom right: result of querying the Crystallography Open Database [30], after searching for structures with elements C, N, O, F, Fe and 6 different elements. A simple double-click allows to load the CIF in FOX, where the molecules are automatically built by analysing the atomic distances. 


\section{Examples}

\subsection{Inorganic Structure—Rigid Body and Orientation Disorder}

Solving crystal structures containing large rigid fragments, such as molecules or complex ions, is simplified in FOX by easy creation of the fragments available as pre-programmed objects, or by importing fragments from a file containing a Z-matrix description. Good examples of inorganic structures are metal borohydrides and closo-boranes studied as hydrogen storage materials and solid electrolytes [31].

Monoclinic $\mathrm{K}_{3} \mathrm{BH}_{4} \mathrm{~B}_{12} \mathrm{H}_{12}$ is an inorganic crystal structure containing rigid complex anions $\mathrm{BH}_{4}{ }^{-}$and $\mathrm{B}_{12} \mathrm{H}_{12}{ }^{2-}$ [32]. It was solved from synchrotron powder diffraction data in the space group PC using three independent potassium atoms, one borohydride $\left(\mathrm{BH}_{4}{ }^{-}\right)$and one closo-borane $\left(\mathrm{B}_{12} \mathrm{H}_{12}{ }^{2-}\right.$ ) complex anion (Figure 4$)$. While the tetrahedron of the borohydride is available in FOX as a pre-programmed object, the closo-borane was imported from the Z-matrix. The structural model was then verified by DFT calculations and its symmetry corrected to $P 2 / c$. The overlooked 2-fold axis is due to the weak contribution of hydrogen atoms of the borohydride to the $X$-ray powder diffraction pattern, as it was broken only by the borohydride orientation.

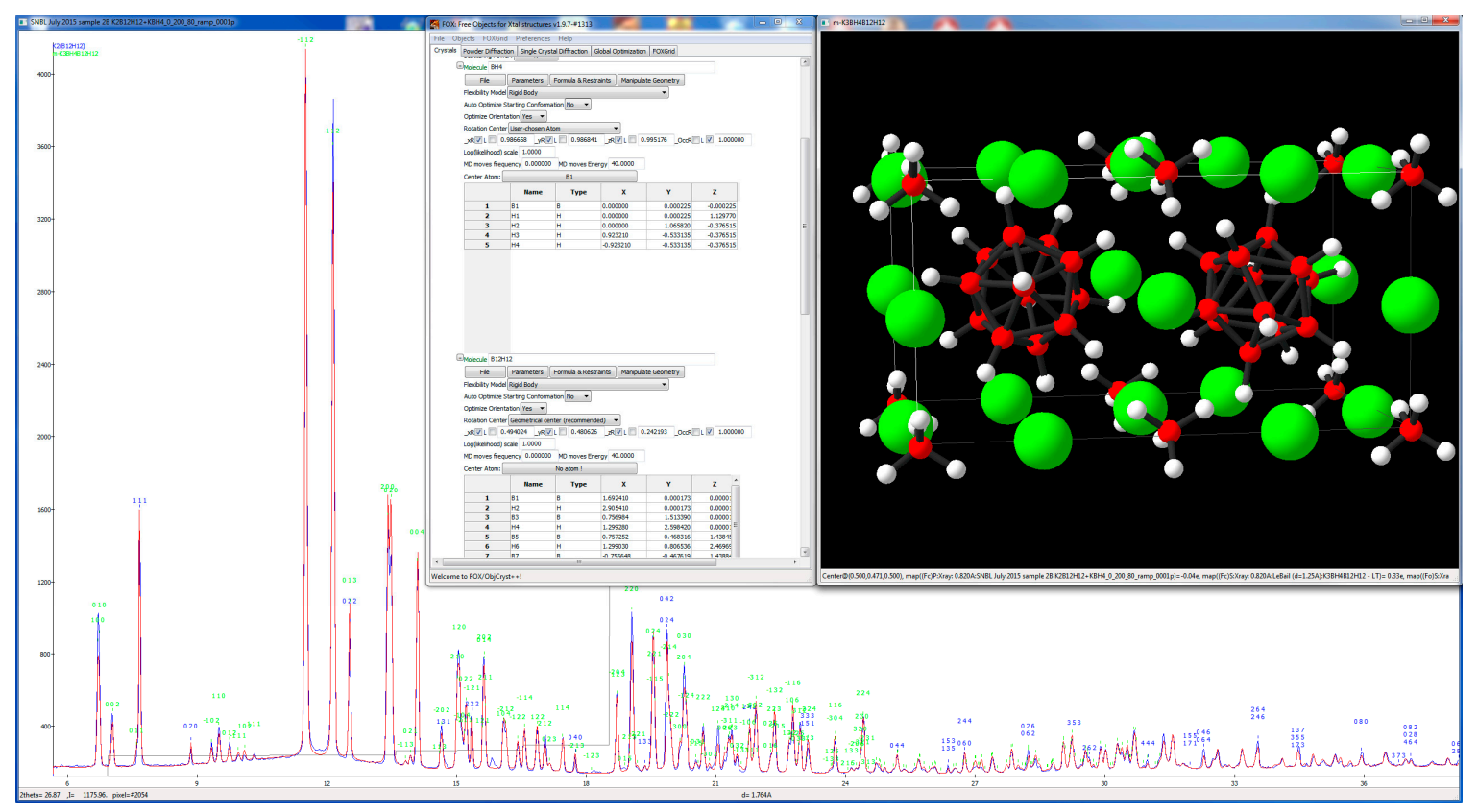

Figure 4. Monoclinic $\mathrm{K}_{3} \mathrm{BH}_{4} \mathrm{~B}_{12} \mathrm{H}_{12}$, an example of an inorganic structure $(P 2 / c, a=7.0497(2)$, $b=6.9917(2), c=13.4192(3) \AA, \beta=94.508(1)^{\circ}$ at $\left.\mathrm{RT}\right)$ with rigid complex anions $\mathrm{BH}_{4}{ }^{-}$and $\mathrm{B}_{12} \mathrm{H}_{12}{ }^{2-}$ [32]. Synchrotron powder diffraction data (SNBL at the ESRF Grenoble, $\lambda=0.81984 \AA$ ), $\mathrm{K}_{3} \mathrm{BH}_{4} \mathrm{~B}_{12} \mathrm{H}_{12}$ (peaks labelled in green), unreacted $\mathrm{K}_{2} \mathrm{~B}_{12} \mathrm{H}_{12}$ (peaks labelled in blue), potassium (green), boron (red), hydrogen (white).

Modelling of the orientation disorder of rigid fragments (closo-borane in our example where the disorder is dynamical and supporting cation mobility) is simplified in FOX by Dynamical Occupancy Correction (DOC) [1], which also takes into account the point symmetry of the Wyckoff site where the fragment is localized. The distance between the boron or hydrogen atoms in the disordered closo-borane stays below the activation limit of DOC $(1 \AA)$, providing the correct scattering density of the fragment in any orientation. 


\subsection{Organic Structure—Molecule with Flexible Cycle}

An interesting example where the usage of the MD approach was crucial is the crystal structure of the 17-membered cyclic Schiff base [33]. According to the volume of the orthorhombic unit cell $\left(a=4.71769(3), b=19.1524(2), c=22.9418(2) \AA, V=2072.90(3) \AA^{3}\right)$, and the fact that the systematic extinctions satisfying $P 22_{1} 2_{1} 2_{1}$ space group, the asymmetric part of the unit cell contains only one molecule of the Schiff base $(Z=4)$ with 21 DoF. The solution should be easily found in a couple of minutes or a few hours. However, the determination of the crystal structure by using the default setting of the global optimization algorithm was not giving any satisfactory results after 10 tested runs, each with $5 \times 10^{6}$ trials (approximately 24 min per run). On the other hand, all 10 runs of the MD approach (move's frequency was set to 0.05 and amplitude of the move's energy was set to 60 -small distortion of the molecule), where each run tested $5 \times 10^{6}$ trials (approximately $50 \mathrm{~min}$ per run), resulted in all 10 giving promising results with low values for cost functions, and with reasonable and similar conformations of the Schiff base.

Comparison of behaviour of the CFs (Cost Functions) during the structure solution process revealed a problem with the convergence of the CF during the default run; see Figure 5. Every single run of the MD approach resulted in some independent solution, and the evolution of the CF behaved as expected for the global optimization process. However, all results of the classic approach were only a slight improvement on previous runs, which is also obvious from the comparison of molecular shapes of all 10 results with the correct one obtained by Rietveld refinement; see Figure 6. One may say that, in the case of the default run, the near-correct solution was finally reached in the last run, which means, overall, after $50 \times 10^{6}$ trials.
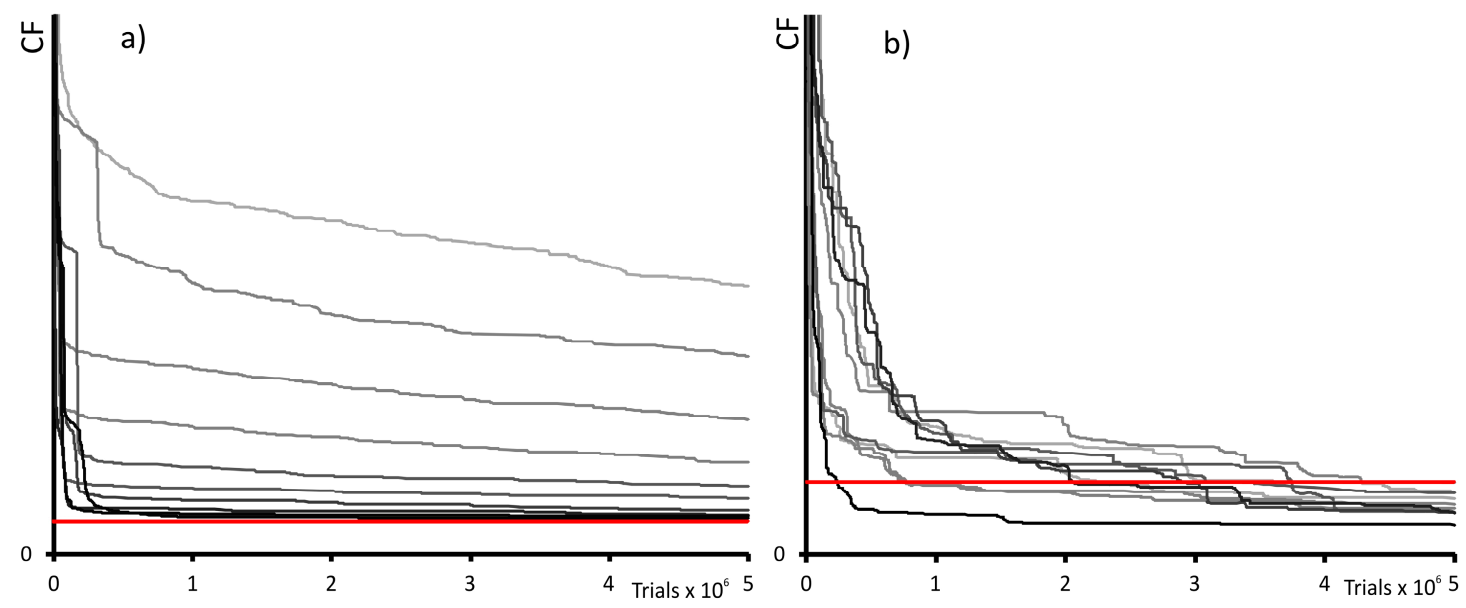

Figure 5. Evolution of the CF (Cost Function) during the 10 runs a) when the default setting of the optimization algorithm was used and b) when the MD approach was used. Evolution of CF of each run is drawn with different shade of grey, starting from light grey for the first run, and ending with black for the final, 10th, run. Both graphs are zoomed to see more detail, and the red line shows the value of the $\mathrm{CF}$ at $4.26 \times 10^{6}$, which corresponds to the highest resulting CF value in the MD result list, and illustrates the "scale" between left and right graphs. 
a)

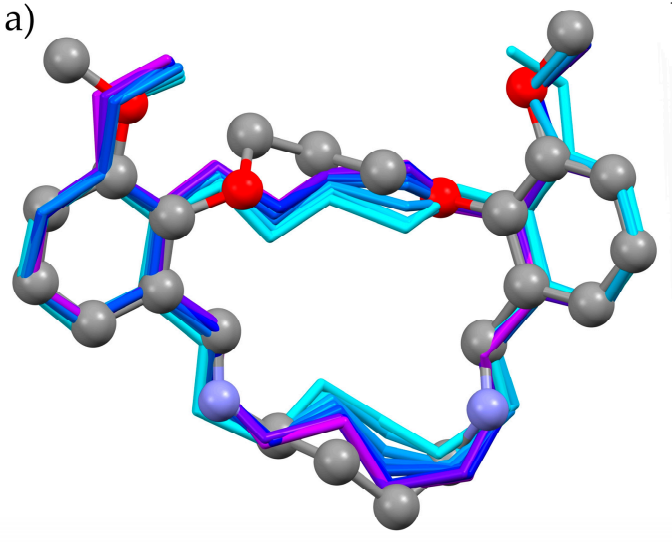

b)

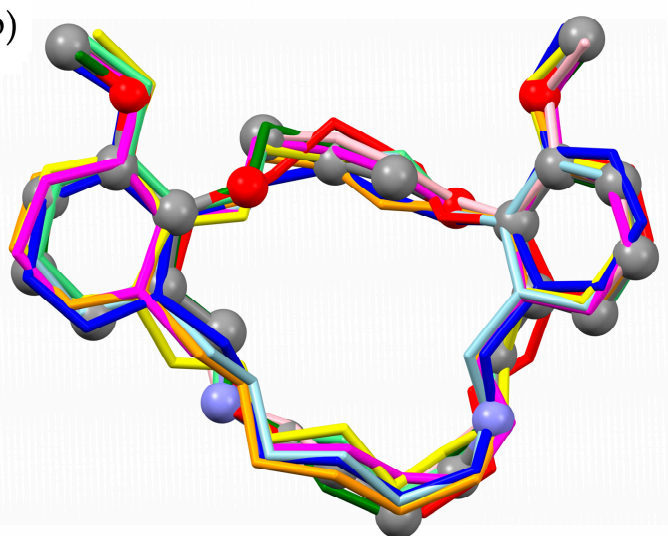

Figure 6. (a) Overlay of results found by the default setting of the parallel tempering algorithm without MD approach. Evolution of the conformation towards the correct one is clearly visible from the changing colour of the first (light blue) to the last (purple) result. (b) Overlay of results found by the MD approach. One-coloured stick styles represent results suggested by the described optimization process combined with MD moves. The picture shows that atomic positions suggested by the optimization method are distributed around the correct atomic positions. Ball style with different atomic colours represents the published solution in both parts of the picture.

\section{Availability of FOX}

FOX, 'Free Objects for Xtallography' is open-source software, available for free for Linux, Windows and MacOS from http://fox.vincefn.net. The source code is available directly from Sourceforge (https:/ / sourceforge.net/projects/objcryst/) and GitHub (https:/ / github.com/vincefn/ objcryst/releases). The underlying library can also be used with Python using the pyobjcryst project (http://www.diffpy.org/pyobjcryst/).

Supplementary Materials: Powder diffraction data, FOX files and files containing Z-matrix description of molecules and rigid bodies are available as ZIP files at: www.mdpi.com/2073-4352/7/10/322/s1.

Acknowledgments: The authors are indebted to the numerous FOX users who have supplied examples, discussed new features and reported bugs. We would also like to thank all the code contributions, notably from Brian Toby and Michael Polyakov (display of Fourier maps), Christopher Farrow and Pavol Juháš, Aleksander Zaloga.

Author Contributions: V.F.-N. has written a major part of the text, R.C. has written a part on inorganic structure example, and J.R. and M.H. on organic structure example. All authors has read and finalized the manuscript.

Conflicts of Interest: The authors declare no conflict of interest. The founding sponsors had no role in the design of the study; in the collection, analyses, or interpretation of data; in the writing of the manuscript, and in the decision to publish the results.

\section{References}

1. Favre-Nicolin, V.; Černý, R. FOX, "Free Objects for Crystallography": A modular approach to ab-initio structure determination from powder diffraction. J. Appl. Cryst. 2002, 35, 734-743. [CrossRef]

2. Favre-Nicolin, V.; Černý, R. A better FOX: Using flexible modelling and maximum likelihood to improve direct-space ab initio structure determination from powder diffraction. Z. Kristallogr. 2004, 219, 847-856. [CrossRef]

3. Update on ab-Initio Structure Determination Using FOX. Available online: http:/ / www.iucr.org/_data/ assets/pdf_file/0014/21623/cpd31.pdf (accessed on 24 October 2017).

4. Černý, R.; Favre-Nicolin, V. FOX: A friendly tool to solve nonmolecular structures from powder diffraction. Powder Diffr. 2005, 20, 359-365. [CrossRef]

5. FOX, Free Objects for Xtallography Version 1.7 (November 2005). Available online: http:/ /www.iucr.org/_ data/assets/pdf_file/0003/21657/CPD32.pdf (accessed on 24 October 2017). 
6. Černý, R.; Favre-Nicolin, V.; Rohlícek, J.; Hušák, M.; Matej, Z.; Kužel, R. Expanding FOX: Auto-indexing, grid computing, profile fitting. Comm. Powder Diffr. (IUCr) Newsl. 2007, 35, 16-19.

7. David, W.I.F.; Sivia, D.S. Background estimation using a robust Bayesian analysis. J. Appl. Cryst. 2001, 34, 318-324. [CrossRef]

8. Boultif, A.; Louër, D. Indexing of powder diffraction patterns for low-symmetry lattices by the successive dichotomy method. J. Appl. Cryst. 1991, 24, 987-993. [CrossRef]

9. Boultif, A.; Louër, D. Powder pattern indexing with the dichotomy method. J. Appl. Cryst. 2004, 37, 724-731. [CrossRef]

10. Louër, D.; Boultif, A. Powder pattern indexing and the dichotomy algorithm. Z. Kristallogr. Suppl. 2007, 26, 191-196. [CrossRef]

11. De Wolff, P.M. The definition of the indexing figure of merit M20. J. Appl. Cryst. 1972, 5, 243. [CrossRef]

12. Černý, R.; Filinchuk, Y. Complex inorganic structures from powder diffraction: Case of tetrahydroborates of light metals. Z. Kristallogr. 2011, 226, 882-891. [CrossRef]

13. Le Bail, A. Whole powder pattern decomposition methods and applications: A retrospection. Powder Diffr. 2005, 20, 316-326. [CrossRef]

14. McCusker, L.B.; Von Dreele, R.B.; Cox, D.E.; Louër, D.; Scardi, P. Rietveld refinement guidelines. J. Appl. Cryst. 1999, 32, 36-50. [CrossRef]

15. Markvardsen, A.J.; David, W.I.F.; Johnson, J.C.; Shankland, K. A probabilistic approach to space-group determination from powder diffraction data. Acta Cryst. A 2001, 57, 47-54. [CrossRef]

16. Pitt, M.P. In-situ Powder Diffraction Studies of Metal-Hydrogen Microstructures. Ph.D. Thesis, Griffith University, Nathan, Australia, 2003.

17. Dollase, W.A. Correction of intensities for preferred orientation in powder diffractometry: Application of the March model. J. Appl. Cryst. 1986, 19, 267-272. [CrossRef]

18. Falcioni, M.; Deem, M.W. A Biased Monte Carlo Scheme for Zeolite Structure Solution. J. Chem. Phys. 1999, 110, 1754-1766. [CrossRef]

19. Earl, D.J.; Deem, M.W. Parallel tempering: Theory, applications, and new perspectives. Phys. Chem. Chem. Phys. 2005, 7, 3910-3916. [CrossRef] [PubMed]

20. David, W.I.F.; Shankland, K.; Van de Streek, J.; Pidcock, E.; Motherwell, W.D.S.; Cole, J.C. DASH: A program for crystal structure determination from powder diffraction data. J. Appl. Cryst. 2006, 39, 910-915. [CrossRef]

21. Johnston, J.C.; David, W.I.F.; Markvardsen, A.J.; Shankland, K. A hybrid Monte Carlo method for crystal structure determination from powder diffraction data. Acta Cryst. A 2002, 58, 441-447. [CrossRef]

22. Markvardsen, A.J.; Shankland, K.; David, W.I.F.; Didlick, G. Characterization of a hybrid Monte Carlo search algorithm for structure determination. J. Appl. Cryst. 2005, 38, 107-111. [CrossRef]

23. Turner, G.W.; Tedesco, E.; Harris, K.D.M.; Johnston, R.L.; Kariuki, B.M. Implementation of Lamarckian concepts in a Genetic Algorithm for structure solution from powder diffraction data. Chem. Phys. Lett. 2000, 321, 183-190. [CrossRef]

24. Cernik, R.J.; Cheetham, A.K.; Prout, C.K.; Watkin, D.J.; Wilkinson, A.P.; Willis, B.T.M. The structure of cimetidine $\left(\mathrm{C}_{10} \mathrm{H}_{16} \mathrm{~N}_{6} \mathrm{~S}\right)$ solved from synchrotron-radiation X-ray powder diffraction data. J. Appl. Cryst. 1991, 24, 222-226. [CrossRef]

25. Shankland, K.; Markvardsen, A.J.; Rowlatt, C.; Shankland, N.; David, W.I.F. A benchmark method for global optimization problems in structure determination from powder diffraction data. J. Appl. Cryst. 2010, 43, 401-406. [CrossRef]

26. Fernandes, P.; Shankland, K.; David, W.I.F.; Markvardsen, A.J.; Florence, A.J.; Shankland, N.; Leech, C.K. A differential thermal expansion approach to crystal structure determination from powder diffraction data. J. Appl. Cryst. 2008, 41, 1089-1094. [CrossRef]

27. Griffin, T.A.N.; Shankland, K.; Van de Streek, J.; Cole, J. GDASH: A grid-enabled program for structure solution from powder diffraction data. J. Appl. Cryst. 2009, 42, 356-359. [CrossRef]

28. Spillman, M.J.; Shankland, K.; Williams, A.C.; Cole, J.C. CDASH: A cloud-enabled program for structure solution from powder diffraction data. J. Appl. Cryst. 2015, 48, 2033-2039. [CrossRef]

29. Recent Developments of the Program FULLPROF. Available online: http://www.iucr.org/_data/assets / pdf_file/0019/21628/cpd26.pdf (accessed on 24 October 2017). 
30. Gražulis, S.; Chateigner, D.; Downs, R.T.; Yokochi, A.F.T.; Quirós, M.; Lutterotti, L.; Manakova, E.; Butkus, J.; Moeck, P.; Le Bail, A. Crystallography Open Database-An open-access collection of crystal structures. J. Appl. Cryst. 2009, 42, 726-729. [CrossRef] [PubMed]

31. Paskevicius, M.; Jepsen, L.H.; Schouwink, P.; Černý, R.; Ravnsbaeck, D.; Filinchuk, Y.; Dornheim, M.; Besenbacher, F.; Jensen, T.R. Metal borohydrides and derivatives-Synthesis, structure and properties. Chem. Soc. Rev. 2017, 46, 1565-1634. [CrossRef] [PubMed]

32. Sadikin, Y.; Skoryunov, R.V.; Babanova, O.A.; Soloninin, A.V.; Lodziana, Z.; Brighi, M.; Skripov, A.V.; Černý, R. Anion disorder in $\mathrm{K}_{3} \mathrm{BH}_{4} \mathrm{~B}_{12} \mathrm{H}_{12}$ and its effect on cation mobility. J. Phys. Chem. C 2017, 121, 5503-5514. [CrossRef]

33. Khalaji, A.D.; Ghoran, S.H.; Rohlíček, J.; Dušek, M. Characterization and crystal structure of a 17-membered macrocyclic Schiff base compound MeO-sal-pn-bn. J. Struct. Chem. 2015, 56, 259-265. [CrossRef]

(c) 2017 by the authors. Licensee MDPI, Basel, Switzerland. This article is an open access article distributed under the terms and conditions of the Creative Commons Attribution (CC BY) license (http://creativecommons.org/licenses/by/4.0/). 